\title{
Evaluation of Uncertainty in Dynamic Simulations of Power System Models: The Probabilistic Collocation Method
}

\author{
James R. Hockenberry, Member, IEEE, and Bernard C. Lesieutre, Member, IEEE
}

\begin{abstract}
This paper explores the use of a new technique, the probabilistic collocation method (PCM), to enable the evaluation of uncertainty in power system simulations. The PCM allows the uncertainty in transient behavior of power systems to be studied using only a handful of simulations. The relevant theory is outlined here and simple examples are used to illustrate the application of PCM in a power systems setting. In addition, an index for identification of key uncertain parameters, as well as an example with a more realistic power system, are presented.
\end{abstract}

Index Terms-Power system modeling, power system simulation, uncertainty.

\section{INTRODUCTION}

$\mathbf{T}$ IME-STEP simulation techniques form an important class of tools for power system analysis. They are employed whenever dynamic phenomena are to be studied, from the standalone analysis of individual components or the study of fast electromagnetic transients, to the behavior of large-scale systems over many time scales. (We use the words "time-step simulation" to refer to methods that emulate the dynamic response of a system typically represented by ordinary differential and algebraic equations, and to distinguish from the usage of "simulation" to refer to techniques to evaluate probabilistic problems using Monte Carlo and related methods.) The results of such studies are used to make decisions concerning the structure, tuning, and operation of the system. The constant use and importance of these tools motivates the equally constant research on improving models and simulation algorithms. The contribution of this paper is to demonstrate practical evaluation of uncertainties in power system models using presently available simulation tools. This method will enable detailed uncertainty studies that have been infeasible due to computational limitations. By directly considering uncertainties in the models, the additional information gained will lead to less need for conservative operation of the power grid.

The literature concerning uncertainty analyses in power systems is vast (see the bibliographies in [1]-[7]). The referenced

Manuscript received January 9, 2004.

J. R. Hockenberry was with the Massachusetts Institute of Technology, Cambridge, MA 02139 USA. He is now with Robert Bosch, GmbH, Stuttgart 70049, Germany (e-mail: james.hockenberry@ de.bosch.com).

B. C. Lesieutre was with the Massachusetts Institute of Technology, Cambridge, MA 02139 USA. He is now with the Lawerence Berkeley National Laboratory, Berkeley, CA 94720 USA (e-mail: BCLesieutre@lbl.gov).

Digital Object Identifier 10.1109/TPWRS.2004.831689 books and papers cover a range of topics, many related to statistical reliability studies. With regard to uncertainty studies addressing transient stability, there are fewer papers, and these consider uncertainties related to disturbances and operating conditions [8]-[14]. These do not consider uncertainties in parameter values, and the approaches combine deterministic simulation techniques with stochastic analyses. In some cases [11], [10] the dynamic simulation step is significantly enhanced by the use of Lyapunov-like energy functions. However, these energy functions are based on simplified models of a power system that lack significant detail. While these studies are important in their own right, they do not address the problem we consider and do not design the time-step simulations around the uncertainty characterization.

The specific area of parameter uncertainties in detailed time-domain simulation studies is nearly vacant. This is understandable. Historically, a time-step simulation of a large power system model required lengthy computer runs ( $\gg$ hours). With faster computers these same simulations now can be completed in minutes. (However, there is now new interest in simulating larger and more detailed models which increases the simulation time.) Still, practical techniques for the analysis of uncertainty, such as Monte Carlo and its derivatives, require many sample data obtained from repeated simulations. For example, suppose that 1000 points are necessary, obtained from 1000 time-step simulations, then hours to days of computer time will be required. Furthermore, each such study only represents the analysis of a single event (line outage, generator loss, etc.). Evaluating many contingencies while accounting for uncertainties is prohibitively time intensive. Consequently, such studies are not typically done. The results from nominal simulations are incorporated into design and operation in a conservative manner.

The recent literature on this topic is not completely empty. In addition to our initial limited study [15], Hiskens et al. have used their "trajectory sensitivity" approach to approximate the effect of uncertain parameter values on the outcome of time-step simulations [16]. The method essentially employs an augmented model that includes additional variables to represent the sensitivity of specified state variables to select parameters and initial conditions. The result of the time-step simulation of this larger model yields the nominal trajectory and the sensitivities of the trajectory to the aforementioned parameters. These linear sensitivities then can be used to form a linear model with which to approximate the effect of uncertain parameters in a region about a 
nominal trajectory without the need for further time-consuming (time-domain) simulation.

We would like to advocate in this work a technique developed by researchers who study global climate change. Similar to ours, their time-domain simulation studies require significant computer time and their models include parameters with uncertain values. The so-called probabilistic collocation method (PCM) [17], [18] (later renamed the "deterministic equivalent modeling method) involves a few time-step simulations, the results of which are used to develop a polynomial model that directly maps the uncertain parameter to outcomes of interest. This very reduced-order model can be evaluated quickly to determine the effect of the uncertainty. In their studies, the uncertain parameter may be carbon dioxide emissions and the outcome the average temperature in 2100 . In our studies, the uncertainty may be related to load models or fault time and the outcome may be maximum frequency, angle, and voltage variations over the course of the simulation (among many other possibilities) that can be compared against operational and stability limits imposed on these variables.

This approach is particularly appealing because it allows the use of nonlinear models and the evaluation of complicated output functions (maximum deviations). And, importantly, these studies can be performed using existing commercially-available power system simulation software.

The techniques we discuss are applicable to all studies that rely on dynamic simulations: from fast time-scale EMTP simulations to slower time-scale large-scale system studies. In this paper, we focus on system studies for the purpose of accurately assessing operational limits while considering conditions during which disturbances may lead to voltage and frequency violations. Instead of relying on operational safety margins, which we hope are sufficiently conservative, a planning/operational decision can be based on the probability of exceeding a set of specified limits.

Our work on this topic is summarized in this paper. Here we introduce and evaluate the PCM technique applied to small power system models. We also address the additional challenges associated with the analysis of very large system models containing numerous uncertainties as well as presenting a large system example. A more detailed treatment of some of the topics presented here can be found in [19].

\section{THEORY}

PCM essentially creates polynomial models relating the uncertain parameters of the system to the outputs of interest. The power of the PCM method lies in its ability to select appropriate simulation points to create a polynomial model which has the same moments as a higher order model. Judiciously selected simulations are carried out initially in order to determine the coefficients of this polynomial model. Orthogonal polynomials and Gaussian quadrature integration [20] are used to justify the selection of suitable simulations, so these methods will be discussed before the actual development of PCM is presented. One important property of PCM to note in the development is the independence of the simulations necessary to determine the coefficients of the polynomial model from the output(s) of interest.
The same set of simulations is performed to determine the coefficients of the polynomial models for all of the outputs of interest.

\section{A. Orthogonal Polynomials}

The following function is an inner product on the space of polynomials:

$$
\langle g(x), h(x)\rangle=\int_{\mathcal{A}} f(x) g(x) h(x) d x
$$

where $f(x)$ is any nonnegative weighting function defined everywhere in a connected $\mathcal{A}$. In the context of PCM, $f(x)$ is a probability density function (pdf) describing the uncertainty in a system parameter. This particular inner product is the one used for Gaussian quadrature integration and the probabilistic collocation method.

Given this inner product, a pair of polynomials is said to be orthogonal if their inner product is zero. Further, a set of polynomials $\mathcal{H}$ is said to be orthonormal if and only if the following relationship holds for all $h_{i}(x)$ in $\mathcal{H}$ :

$$
\left\langle h_{i}, h_{j}\right\rangle=\left\{\begin{array}{ll}
1, & \text { if } i=j \\
0, & \text { if } i \neq j
\end{array} .\right.
$$

Given a weighting function $f(x)$, we are interested in a particular orthonormal set, $\mathcal{H}$, of polynomials of increasing order in which $h_{i}$ is a polynomial of order $i$. These polynomials are unique and form a basis for all polynomials. Efficient recursive methods exist for obtaining these polynomials [20].

We omit the proofs, but it can be shown that each $h_{i}$ has exactly $i$ roots and all of the roots are contained in $\mathcal{A}$ [20]. These roots play a pivotal role in the probabilistic collocation method.

\section{B. Gaussian Quadrature Integration}

Gaussian quadrature integration is a numerical integration technique for integrals of the form:

$$
\int_{\mathcal{A}} f(x) g(x) d x
$$

where $g(x)$ is a polynomial and $f(x)$ is a nonnegative weighting function. If $f(x)$ is a pdf, this integral is the expected value of $g(x)$. The main result of Gaussian quadrature integration is the following exact formula for calculating this integral:

$$
\int_{\mathcal{A}} f(x) g(x) d x=\sum_{i=1}^{n} f_{i} g\left(x_{i}\right)
$$

where the $f_{i}$ are constants which only depend on the weighting function $f(x)$ and the $x_{i}$ are constants in the region of integration. The formula is exact for all polynomials $g(x)$ of order less than or equal to $2 n-1$. This result is somewhat surprising. The polynomial $g(x)$ itself could be determined using $2 n$ samples, but only $n$ samples of $g(x)$ are needed to compute the integral.

The $x_{i}$ are computed using the orthonormal polynomial set $\mathcal{H}$, where $f(x)$ is used as the weighting function. The constants $x_{i}$ are the roots of $h_{n}$, which exist and are contained in $\mathcal{A}$. Furthermore, the $x_{i}$ only depend on $f(x)$, since $\mathcal{H}$ only depends on $f(x)$. We show the independence of the $f_{i}$ from $g(x)$ and the correctness of (4) with these $x_{i}$ constructively. 
The polynomials in $\mathcal{H}$ up to and including order $i$ constitute an orthonormal basis for the space of all polynomials of degree less than or equal to $i$. Therefore, a polynomial of order $2 n-$ 1 can be expressed in terms of these orthonormal polynomials using constant coefficients $a_{i}$ and $b_{i}$

$$
\begin{aligned}
g(x)=h_{n}(x)\left(a_{n-1} h_{n-1}(x)+\cdots+a_{0} h_{0}(x)\right) \\
+b_{n-1} h_{n-1}(x)+\cdots+b_{0} h_{0}(x) .
\end{aligned}
$$

If this expression is expanded by multiplying through by $h_{n}(x)$, the result is a sum of $2 \mathrm{n}$ linearly independent polynomials (not necessarily orthogonal), which proves that such a representation is always feasible. Since $h_{0}(x)$ is a constant, the integral is easily determined by orthogonality

$$
\int_{\mathcal{A}} f(x) g(x) d x=b_{0} \int_{\mathcal{A}} f(x) h_{0}(x) d x .
$$

Finally, we build a linear set of equations by evaluating $g(x)$ in (5) at the roots of $h_{n}(x)$

$$
\left[\begin{array}{c}
g\left(x_{1}\right) \\
\vdots \\
g\left(x_{n}\right)
\end{array}\right]=\left[\begin{array}{ccc}
h_{n-1}\left(x_{1}\right) & \cdots & h_{0}\left(x_{1}\right) \\
\vdots & \ddots & \vdots \\
h_{n-1}\left(x_{n}\right) & \cdots & h_{0}\left(x_{n}\right)
\end{array}\right]\left[\begin{array}{c}
b_{n-1} \\
\vdots \\
b_{0}
\end{array}\right] .
$$

By inverting this matrix, we derive an expression for $b_{0}$

$$
\left[\begin{array}{c}
b_{n-1} \\
\vdots \\
b_{0}
\end{array}\right]=\left[\begin{array}{ccc}
h_{n-1}\left(x_{1}\right) & \cdots & h_{0}\left(x_{1}\right) \\
\vdots & \ddots & \vdots \\
h_{n-1}\left(x_{n}\right) & \cdots & h_{0}\left(x_{n}\right)
\end{array}\right]^{-1}\left[\begin{array}{c}
g\left(x_{1}\right) \\
\vdots \\
g\left(x_{n}\right)
\end{array}\right] .
$$

If we define $h_{0}(x)$ to be 1 (which is customary) and assume $f(x)$ is a pdf (which integrates to 1 ), then $b_{0}$ is the desired result

$$
b_{0}=\int_{\mathcal{A}} f(x) g(x) d x=\sum_{i=1}^{n} f_{i} g\left(x_{i}\right) .
$$

The weights $f_{i}$ are given by the last row of the matrix in (8). Since $\mathcal{H}$ is determined solely by $f(x)$, both $x_{i}$ and $f_{i}$ are independent of $g(x)$.

The independence of the $x_{i}$ from the particular polynomial for which we are calculating the integral is analogous to the desired independence between the set of parameter values for which we perform simulations and the particular output variable of interest. One set of $x_{i}$ suffices for all polynomials of order less than or equal to $(2 n-1)$ and one set of simulations is sufficient for all outputs of interest.

\section{Monte Carlo Method}

A brief description of the simple, "brute force" Monte Carlo method for a single uncertain parameter is helpful as a basis of comparison before we discuss PCM.

In its simplest form, the Monte Carlo method is a method of repeated trials. As an example, let $f(x)$ be a pdf describing some uncertain parameter $x$

$$
\begin{aligned}
f(x) & \geq 0, \quad \text { for allx } \\
\int_{\mathcal{A}} f(x) d x & =1 .
\end{aligned}
$$

For some desired output, $g(x)$, which is a function of that parameter the expected value of the output is computed using the following integral:

$$
\int_{\mathcal{A}} f(x) g(x) d x=\mathrm{E}[\mathrm{g}(\mathrm{x})] .
$$

A simplistic Monte Carlo method approximates the integral by generating random numbers using $f(x)$ and then performing a simple average of the resulting answers

$$
\int_{\mathcal{A}} f(x) g(x) d x \approx \frac{1}{N} \sum_{i=1}^{N} g\left(\hat{x}_{i}\right)
$$

where the $\hat{x}_{i}$ are generated according to $f(x)$ using a pseudorandom number generator. Given sufficiently large $N$, this method is guaranteed to converge to the actual expected value as a direct result of the law of large numbers [21]. Unfortunately, the required $N$ may be quite large. Since all of the moments of $g(x)$ (e.g., the variance) are expected values, the law of large numbers applies to these statistics as well under the Monte Carlo method.

The size of $N$ is dependent on the variance of the output variable. A larger variance means a larger number of simulations are required. Variance reduction techniques reduce the size of the required $N$ for a particular output by modifying the system and pseudorandom numbers in such a way that the expected value of the desired output is accurately generated but with a significantly reduced variance. However, these methods have the major drawback that a new set of simulations are then necessary for each output variable and each moment, since only the expected value of one output is accurately computed.

\section{PCM-Single Uncertain Parameter}

The probabilistic collocation method is a polynomial modeling technique; the desired output is described as a polynomial in the uncertain parameter of the system. After this model is identified, a standard, simplistic Monte Carlo method can be applied to the polynomial model. The problem with (13) is that each $g\left(x_{i}\right)$ is computationally expensive, since it represents a separate simulation of the power system. If this function $g(x)$ can be modeled reasonably accurately by a polynomial $\hat{g}(x)$, an essentially unlimited number of samples $\hat{g}\left(x_{i}\right)$ can be computed because no simulations are involved once the polynomial has been identified. The only simulations necessary are those to identify the polynomial. If the same simulations can be used to identify the polynomials for all outputs of interest, the number of simulations is very limited in contrast to variance reduction techniques. Therein lies the power of the probabilistic collocation method.

Concretely, let $x$ be the uncertain parameter, $f(x)$ be the pdf describing this parameter and $g(x)$ be the output of interest. PCM creates a polynomial model of the form

$$
\hat{g}(x)=g_{0}^{\prime}+g_{1}^{\prime} x+\cdots+g_{n}^{\prime} x^{n-1}
$$

where the $g_{i}^{\prime}$ are constants. The model parameters could be identified by performing many simulations using various $x_{i}$ and applying a least squares algorithm. However, we are trying to per- 
form an absolute minimal number of simulations, since each simulation is expensive in terms of time.

Gaussian quadrature integration says that if this polynomial is fit using exactly the right simulations, the expected value of $\hat{g}$ is identical to the expected value of $g$ as long as $g$ is a polynomial of order $2 n-1$ or less. PCM fits the polynomial model with simulations of the system at the $x_{i}$ indicated by Gaussian quadrature integration. If a high-order polynomial is a reasonable model for the relationship between uncertain parameter and output of interest, PCM yields extremely good results for the expected value. Not surprisingly, the higher order moments are also well approximated though a firm theoretical basis is lacking.

Following this line of logic, let us represent $\hat{g}(x)$ using the polynomials in $\mathcal{H}$ without loss of generality, where $\hat{g}(x)$ is of order $(n-1)$

$$
\hat{g}(x)=g_{0} h_{0}(x)+g_{1} h_{1}(x)+\cdots+g_{n-1} h_{n-1}(x)
$$

The following linear system of equations can be solved to determine the $g_{i}$ :

$$
\left[\begin{array}{c}
g\left(x_{1}\right) \\
\vdots \\
g\left(x_{n}\right)
\end{array}\right]=\left[\begin{array}{ccc}
h_{n-1}\left(x_{1}\right) & \cdots & h_{0}\left(x_{1}\right) \\
\vdots & \ddots & \vdots \\
h_{n-1}\left(x_{n}\right) & \cdots & h_{0}\left(x_{n}\right)
\end{array}\right]\left[\begin{array}{c}
g_{n-1} \\
\vdots \\
g_{0}
\end{array}\right]
$$

where the $x_{i}$ are chosen to be the roots of $h_{n}(x)$ [to exploit the benefits of Guassian quadrature integration explored in (5)-(9)]. If $h_{0}(x)=1$, the expected value is simply $g_{0}$ and no further calculations are necessary. Similar relationships exist for higher order moments of $g(x)$. For example

$$
\sigma_{g(x)}^{2}=\sum_{i=1}^{n-1} g_{i}^{2}
$$

In general, this approach is heuristic. We cannot usually guarantee that the actual relationship between the uncertain parameter and the output of interest is exactly a polynomial. In addition, we may be interested in further statistical information about the output, not just its moments. As previously noted, higher order moments are also expected values, so the arguments for the expected value hold just as well for the variance, although a higher order polynomial model may be required, but further statistical information, such as the exact probability that the output lies in some interval, may not be accurate. PCM is designed to calculate the coefficients of the polynomial model with the bare minimal number of simulations while also trying to reproduce the moments of the output with high fidelity by modeling the polynomial particularly well in the regions that are more probable. Given that, one could also expect that the PCM model would perform well when used to compute the probability of events in the high probability region of the output.

\section{SIMPLE ILLUSTRATIVE EXAMPLES}

\section{A. RC Circuit}

To illustrate the probabilistic collocation method, a simple first-order circuit, such as the one shown in Fig. 1, may be helpful because of its familiarity and its tractability to analytical
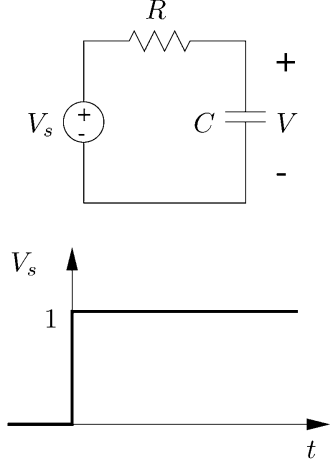

Fig. 1. Simple RC circuit with voltage step input.

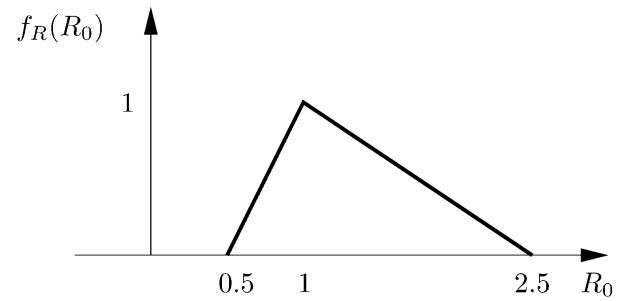

Fig. 2. Pdf for resistance $R$.

techniques. The response voltage is described by a first-order differential equation

$$
\dot{V}=-\frac{V}{\mathrm{RC}}+\frac{V_{s}}{\mathrm{RC}} .
$$

Given zero initial conditions and the step input shown, the output is a familiar exponential response

$$
V(t)=1-e^{-t / \mathrm{RC}} .
$$

For this example, the resistance is treated as uncertain and the capacitance is treated as if it were known exactly. The voltage at time $C / 10$ is chosen as the output of interest $g$

$$
g(R)=V(C / 10)=1-e^{-1 / 10 R}
$$

Before any uncertainty analysis can be performed, a description of the parameter uncertainty must be available. We use a somewhat unusual probability density function to describe the uncertainty in $R$ to demonstrate that the probabilistic collocation method can accommodate any desired pdf

$$
f_{R}\left(R_{0}\right)=\left\{\begin{array}{ll}
2 R_{0}-1, & \frac{1}{2} \leq R_{0}<1 \\
-\frac{2}{3} R_{0}+\frac{5}{3}, & 1 \leq R_{0} \leq \frac{5}{2}
\end{array} .\right.
$$

This pdf is shown graphically in Fig. 2. For completeness, we compute the expected value and variance of $R$

$$
\begin{aligned}
\mathrm{E}[R] & =\frac{4}{3} \\
\sigma_{R}^{2} & =\frac{13}{72}=0.1806 .
\end{aligned}
$$

Since an algebraic expression for $g$ as a function of $R$ is available, the pdf for $g$ can be derived directly, along with its associated moments. The expected value, variance and standard deviation of $g$ are summarized in Table I. 
TABLE I

EXPeCted VAlue, VARIANCE, AND STANDARD DEVIATION FoR RC-CIRCUIT EXAMPLE

\begin{tabular}{c||c|c|c}
\hline & $E[g]$ & $\sigma_{g}^{2}$ & $\sigma_{g}$ \\
\hline \hline Analytical & 0.0797 & 0.000660 & 0.0257 \\
\hline PCM - Linear & 0.0787 & 0.000462 & 0.0215 \\
\hline PCM - Quadratic & 0.0795 & 0.000618 & 0.0249 \\
\hline Taylor - R=1 & 0.0650 & 0.00148 & 0.0385 \\
\hline Taylor - R=4/3 & 0.0723 & 0.000492 & 0.0222 \\
\hline
\end{tabular}

At this point, we would like to demonstrate how to apply PCM to this problem. In normal practice, one would not use PCM in such a situation, since the relationship between the uncertain parameter and the output of interest is known analytically, but having the exact answer for comparison purposes is helpful pedagogically. The first step is to obtain the first few orthogonal polynomials based on $f_{R}\left(R_{0}\right)$ (see [20] for a straightforward recursive algorithm for the construction of orthogonal polynomials)

$h_{0}(R)=1$

$h_{1}(R)=\frac{12}{\sqrt{26}}\left(R-\frac{4}{3}\right)$

$h_{2}(R)=\frac{1560}{\sqrt{96915}}\left(R^{2}-\frac{37}{13} R+\frac{191}{104}\right)$

$h_{3}(R)=\frac{39760}{\sqrt{15174901}}\left(R^{3}-\frac{2157}{497} R^{2}+\frac{29163}{4970} R-\frac{6087}{2485}\right)$.

The roots of $h_{2}(R)$ yield two values of $R$, which we can use to develop a linear approximation of the relationship between $g$ and $R$. The roots of $h_{2}(R)$ and the resulting values of $g$ are as follows:

$$
\begin{aligned}
& R=1.857 \rightarrow g(R)=0.0524 \\
& R=0.989 \rightarrow g(R)=0.0962 .
\end{aligned}
$$

These two points are sufficient to uniquely define a line, which is the linear PCM approximate model

$$
\begin{aligned}
\tilde{g}(R) & =(-0.0505) R+0.146 \\
& =-0.0215 h_{1}(R)+0.0787 h_{0}(R) .
\end{aligned}
$$

As noted earlier, the expected value, variance and standard deviation are directly available from the coefficients and the results are shown in Table I. The accuracy of these results is startling considering that they are based on a linear approximation and that approximation was created using two points selected based on the pdf for $R$ and not based on the relationship between $g$ and $R$.

As a basis of comparison, we also examine another standard way to obtain a linear approximation of $g(R)$, namely, a Taylor series approach. Instead of creating a line using two widely separated values of $R$, we select a nominal value for $R$ and another value of $R$ close to the nominal value, which results in a line tangential to the actual relationship between $g$ and $R$ at that point; in other words, the approximation is local. One might select $R=1$ for the nominal value, since the pdf for $R$ is max-

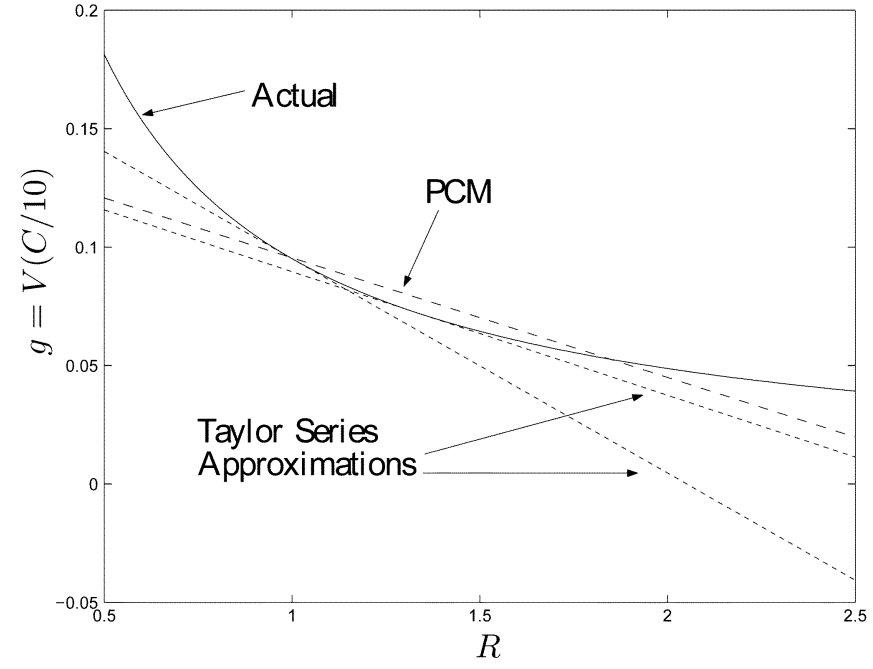

Fig. 3. Comparison of linear approximations using PCM and Taylor series approximation.

imal there. Another good choice is $R=4 / 3$, since that is the expected value of $R$. The PCM linear model as well as these two linear approximations are shown in Fig. 3.

As Table I shows, the PCM results compare favorably with those based on the Taylor series approximations. The results using $R=1$ as the nominal point are uniformly poor since this approximation diverges substantially from the actual $g$ for larger values of $R$. The approximation based on a nominal value of $R=4 / 3$ yields a comparable variance and standard deviation to that using PCM since their slopes are nearly identical. But, the expected value as computed using PCM is more accurate since the Taylor series approximation in this example always lies below the actual graph of $g$; the PCM model is not limited to being tangential to $g$.

One can also create higher order polynomial approximations using PCM. To create a quadratic model, the roots of $h_{3}(R)$ are used to find the coefficients. The roots of $h_{3}$ and the corresponding values of $g(R)$ are as follows:

$$
\begin{aligned}
& R=2.106 \rightarrow g(R)=0.0464 \\
& R=1.407 \rightarrow g(R)=0.0686 \\
& R=0.826 \rightarrow g(R)=0.1140 .
\end{aligned}
$$

The quadratic PCM model is as follows:

$$
\begin{aligned}
\tilde{g}(R) & =0.0072 h_{2}(R)-0.0238 h_{1}(R)+0.0795 h_{0}(R) \\
& =(0.0362) R^{2}-(0.159) R+0.221 .
\end{aligned}
$$

The moments can be computed directly from the coefficients, just as for the linear model, and the results are in Table I. The quadratic PCM model is able to reproduce the actual moments almost exactly.

\section{B. Multiple Machine Power System}

A power system with multiple machines has richer behavior than the previously presented example and we close this section with such a system. The power system model comprises a two-area system adapted from [22] and shown in Fig. 4. We explore the amount of power which can be transferred between the 


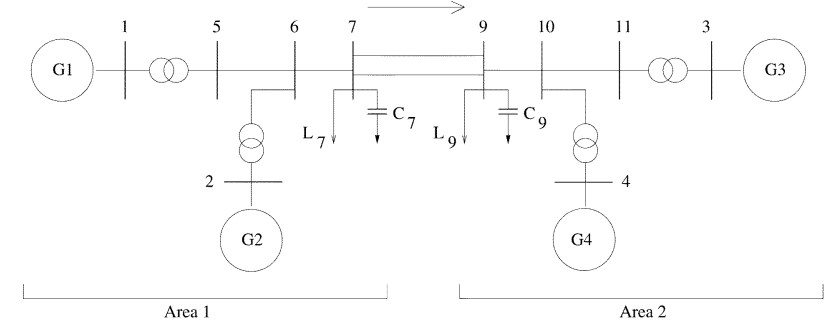

Fig. 4. A small multiple machine power system.

TABLE II

COMPARISON OF PCM AND MONTE CARLO Method

\begin{tabular}{l||l|l}
\hline & Monte Carlo & $\begin{array}{l}\text { PCM, quadratic } \\
\text { 3 pts. }\end{array}$ \\
\hline \hline $\mathrm{E}\left[\mathrm{V}_{\min }\right]$ & 0.913 & 0.913 \\
\hline $\mathrm{SD}\left[\mathrm{V}_{\min }\right]$ & 0.00830 & 0.00833 \\
\hline Prob. of Violation & $7.82 \%$ & $8.92 \%$ \\
\hline
\end{tabular}

two areas. A power transfer is deemed unacceptable if voltage or frequency limits are exceeded during the transient following a fault and reset on one of the two lines connecting buses 7 and 9. If Areas 1 and 2 each represent a utility, we are attempting to determine the maximum amount of power which can safely be transferred from one utility to the other.

At a power transfer of $450 \mathrm{MW}$, the minimum voltage limit is violated during the post-fault transient, while a 400-MW transfer is acceptable. The question we seek to answer is whether the 400-MW transfer scenario is acceptable for all reasonable parameter variations of a single parameter within the model and, if it is not, what is the likelihood that a violation occurs.

For this study, one of the load parameters is modeled as uncertain. A standard real power load model is used in this example, namely

$$
P=P_{0}|V|^{\alpha} .
$$

Here we assume that $\alpha$ is uniformly distributed between 0.75 and 2.0. The load parameter is varied simultaneously and identically at each of the two load locations.

In all cases, when the system transient is unacceptable, the minimum voltage constraint is violated in this system. Therefore, we concentrate on three quantities in our study: the probability of violation, the expected value of the minimum system voltage during the transient, and the standard deviation of the minimum system voltage during the transient. A comparison of the results of our studies using both Monte Carlo methods and the probabilistic collocation method is presented in Table II.

As the table shows, the results using PCM are very good considering that they require only three simulations. As expected, the moments can be calculated to a high degree of accuracy. Even the probability of violation, which is a low probability event, is reasonably approximated. To verify our results, 7000 Monte Carlo simulations are used as a basis of comparison. Realistically, "only" about 1500 Monte Carlo simulations are actually necessary to obtain results with comparable accuracy to those obtained using the probabilistic collocation method.

To complete the discussion of the probability of violation, Table III presents results obtained using progressively higher
TABLE III

COMPARISON OF POLYNOMIAL ORDERS

\begin{tabular}{c||c|c|c}
\hline Order & $\mathrm{E}\left[\mathrm{V}_{\min }\right]$ & $\mathrm{SD}\left[\mathrm{V}_{\min }\right]$ & Probability \\
\hline \hline Linear & 0.91300 & 0.00800 & $3.00 \%$ \\
\hline Quadratic & 0.91333 & 0.00833 & $8.92 \%$ \\
\hline 3rd Order & 0.91346 & 0.00834 & $8.83 \%$ \\
\hline 4th Order & 0.91333 & 0.00831 & $8.90 \%$ \\
\hline 5th Order & 0.91336 & 0.00838 & $8.08 \%$ \\
\hline 6th Order & 0.91321 & 0.00832 & $8.06 \%$ \\
\hline 7th Order & 0.91328 & 0.00825 & $7.86 \%$ \\
\hline
\end{tabular}

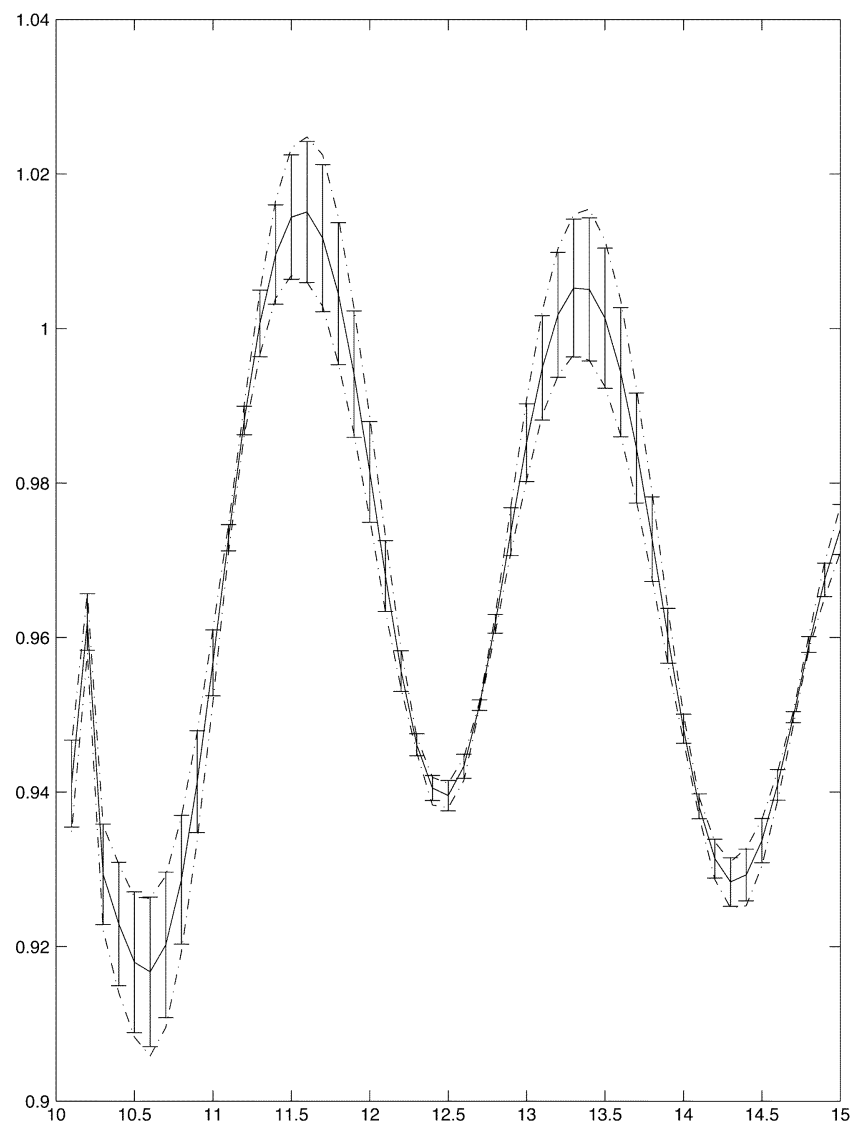

Fig. 5. The voltage at Bus 7 following the fault with error bars.

order polynomial representations. Determining the appropriate order of the polynomial model for a given problem remains an unresolved issue.

Another goal is to perform such studies on time-varying outputs of the system. To illustrate, we take this same example and study the voltage at Bus 7 after the fault is cleared. In this case, we seek something akin to error bars for the simulation. To accomplish this, we plot the expected value of the voltage at each time point along with two additional curves at plus and minus one standard deviation from the mean. Fig. 5 summarizes the results using both brute force Monte Carlo and PCM. The solid line represents the expected value of the voltage as calculated by either a Monte Carlo method or PCM (they yield essentially identical results here). The error bars represent \pm 1 standard deviation from the mean as calculated using a brute force Monte Carlo method. The "dotted-dashed" lines represent \pm 1 standard deviation from the mean as calculated using PCM. The PCM results require three simulations of the system, while the Monte 
Carlo results require 1500 . The correspondence between the two methods is striking, as is the considerable computational savings of PCM.

On a final note, we observe that such time-varying simulation studies are impossible to produce using variance reduction techniques. We individually calculate the expected value and standard deviation at each of 100 time-sample points in order to produce such a plot. One single importance sampling distribution cannot be found which yields good results for all of these different "output variables". On the other hand, the exact same three system simulations are used to create a different polynomial for each of the time points with the probabilistic collocation method. Additional savings are obtained if one is only interested in the mean and standard deviation, since they are directly available from the coefficients of the PCM model.

\section{LARGE POWER SYSTEMS}

We very briefly discuss some of the issues presented by large power systems and present a large power system example. The interested reader is referred to [19] for a more thorough presentation.

\section{A. Parameter Uncertainty Priorization}

In the previous sections, PCM was applied to problems with only one uncertain parameter. In the case of a single uncertain parameter, the number of simulations necessary to fit the model is equal to one plus the order of the polynomial. For multiple uncertain parameters, the required number of simulations grows quickly. Even for a quadratic approximation, a model with $n$ uncertain parameters requires

$$
1+2 n+\left(\begin{array}{l}
n \\
2
\end{array}\right)
$$

simulations. To retain the advantages of PCM, $n$ must be relatively small.

Our approach is to use eigenvalue sensitivities. The basic technique used is inspired by recent work on the identification of key load dynamics for system damping in power systems [23]-[27]. While this research encompasses two separate approaches (which are compared in [27]), eigenvalue sensitivities are at the core of the work described by both research groups. Concretely, we evaluate the following expression for each uncertain parameter $y$ in the system:

$$
\sum_{i=1}^{n}\left|\frac{\partial \lambda_{i}}{\partial y}\right|\left|\frac{y}{\lambda_{i}}\right|\left|\frac{\sigma_{y}}{\mathrm{E}[y]}\right|
$$

where the first term in the product is the absolute sensitivity of the eigenvalue to parameter $y$, the second term is the quotient of the nominal value of the parameter and the nominal value of the eigenvalue, and the last term is the standard deviation of the parameter divided by its expected value. (The ever-present zero-eigenvalue is not considered as it does not change with parameter variation.) This measure is a reasonable way to combine all of the sensitivities into one single scalar quantity, which can be computed quickly. The quantity is also unitless and based on both the relative sensitivity of the eigenvalues and the uncertainty in the parameter (as measured by standard deviation).
TABLE IV

Size OF THE "LARGE" SyStem Before AND AfTER REDUCtion

\begin{tabular}{c|c|c}
\hline & Unreduced & Reduced \\
\hline \# of Gens./Loads & $262 / 670$ & $16 / 63$ \\
\# of Buses Removed & - & 800 \\
\# of Lines Removed/Created & - & $1209 / 282$ \\
\# of Injectors & - & 49 \\
Simulation Time (seconds) & 224 & 34 \\
\hline
\end{tabular}

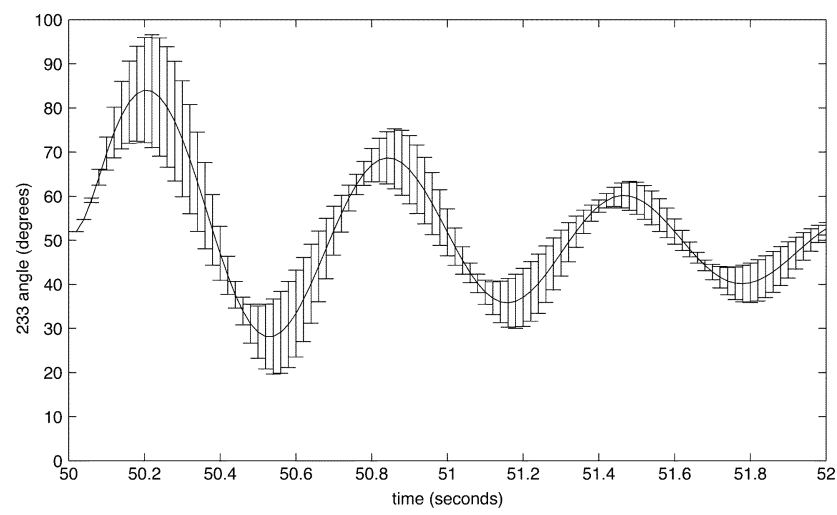

Fig. 6. Uncertainty in generator 233 angle.

\section{B. Large Power-System Example}

A portion of a dynamic model which describes a large portion of the western U.S. was adapted for the studies here. Unfortunately, the data had to be modified from its original form because of problems converting it to conform to the EUROSTAG format. Though the system studied is not necessarily an accurate representation of the original system, the system is nevertheless interesting and realistic. In addition, we applied a model reduction technique to the system; for this paper, we used synchronic modal equivalencing (SME) [28]-[32]. The size of the network before and after reduction is summarized in Table IV.

The event of interest is a short circuit between nodes 234-448 after $50 \mathrm{~s}$, which is cleared $0.1 \mathrm{~s}$ later; this line is the only direct connection between two groups of generators, and, consequently, faulting the line has a substantial effect on the system.

We have not yet considered uncertainties in parameters which are intrinsic to the simulation event itself. A good example of such a parameter in this particular case is the length of time until the fault is cleared. Instead of using a nominal clearing time of $0.1 \mathrm{~s}$, we assume that the clearing time is uniformly distributed between 0.0333 and $0.1 \mathrm{~s}$. For this study, a thirdorder polynomial is used.

Two outputs of interest are chosen: the speed and angle of generator 233. The results are shown in Fig. 6 and 7. In each case, the diagram shows the results of the uncertainty analysis; the solid line is the expected value and the error bars are \pm 1 standard deviation. These results are the result of a PCM analysis using the reduced model to fit the polynomial.

We stress here that these results are obtained with a minimum of computational effort. The time required for the identification and analysis of the polynomial model (including finding moments) is on the order of seconds. We summarize the time savings in Table V.

The first column represents using PCM with model reduction. The second column is similar but without model reduction. (In 


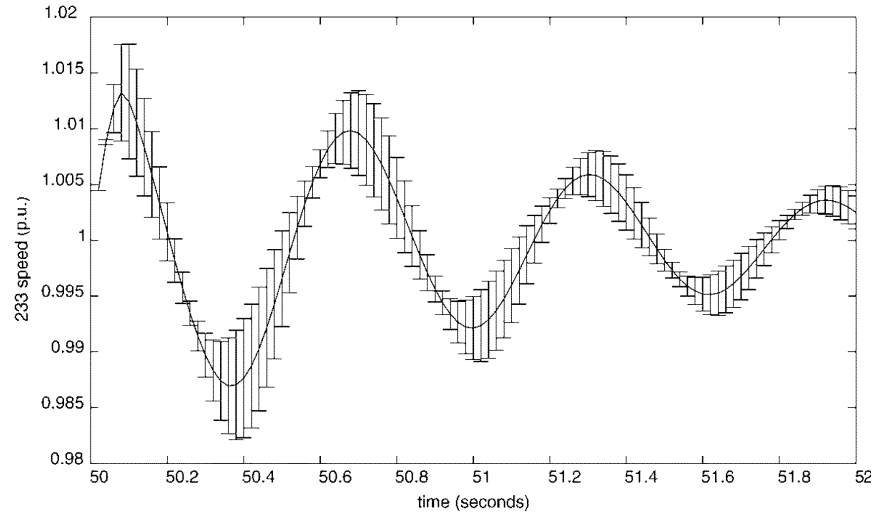

Fig. 7. Uncertainty in generator 233 speed.

TABLE $\mathrm{V}$

Estimated Time-Savings Using the Described Methods

\begin{tabular}{c|c|c|c}
\hline & Our Method & Only PCM & M. C. \\
\hline Reduction Time & $8 \mathrm{~min}$ & - & - \\
\# of Sim. & 4 & 4 & 1000 \\
Time per Sim. & $30 \mathrm{sec}$ & $3 \mathrm{~min}$ & $3 \mathrm{~min}$ \\
Total Time & $\mathbf{1 0 ~} \mathrm{min}$ & $\mathbf{1 2} \mathrm{min}$ & 2 days \\
\hline
\end{tabular}

this case with only one uncertain parameter, model reduction is not strikingly beneficial. We include it only for completeness). The third column is a brute force Monte Carlo approach using the full model. The number of simulations for the Monte Carlo method is a very conservative estimate. The true number of required simulations could easily be several orders of magnitude larger. The time savings using PCM are striking.

\section{CONCLUSION}

The probabilistic collocation method allows one to study the uncertainty in particular outputs, sets of outputs or even transients of a system with a mere handful of simulations. The method relies on polynomial models of the relationship between the uncertain parameter in the system and the outputs of interest. The same set of simulations are used to fit all of the polynomial models for a particular simulation, so the method is more flexible than more traditional time-saving methods, such as variance reduction techniques. The polynomial models are created based on very carefully selected simulations and are not local approximations, as is the case for Taylor series expansion approximations.

The larger system example demonstrates the time savings and enabling aspect of PCM in conjunction with the associated methods. The results presented here would take months to gather using other standard methods.

We believe that this method shows great promise. We hope that it encourages interest in the study of uncertainty in power systems simulations and at the same time enables more sophisticated analyses of uncertainty without prohibitively expensive expenditures of time and resources.

\section{ACKNOWLEDGMENT}

The authors would like to thank the Grainger Foundation for their generous support of this work. The authors would also like to thank C. Taylor of the Bonneville Power Administration for access to large-scale power system data.

\section{REFERENCES}

[1] R. N. Allan, R. Billinton, A. M. Breipohl, and C. H. Grigg, "Bibliography on the application of probability methods in power system reliability evaluation 1992-1996," IEEE Trans. Power Syst., vol. 14, pp. 51-57, Feb. 1999.

[2] — , "Bibliography on the application of probabilitiy methods in power system reliability evaluation 1987-1991," IEEE Trans. Power Syst., vol. 9, pp. 41-49, Feb. 1994

[3] R. N. Allan, R. Billinton, S. M. Shahidehpour, and C. Singh, "Bibliography on the application of probability methods in power system reliability evaluation 1982-1987," IEEE Trans. Power Syst., vol. 3, pp. 1555-1564, Nov. 1988.

[4] R. N. Allan, R. Billinton, and S. H. Lee, "Bibliography on the application of probability methods in power system reliability evaluation 1977-1982," IEEE Trans. Power App. Syst., vol. PAS-103, pp. 275-282, Feb. 1984.

[5] IEEE Subcommittee on the Application of Probability Methods, "Bibliography on the application of probability methods in power system reliability evaluation 1971-1977," IEEE Trans. Power App. Syst., vol. PAS-97, pp. 2235-2242, 1978.

[6] R. Billinton, "Bibliography on the application of probability methods in power system reliability evaluation," IEEE Trans. Power App. Syst., vol. PAS-91, pp. 649-660, Mar./Apr. 1972.

[7] M. T. Schilling, A. M. L. da Silva, R. Billinton, and M. A. El-Kady, "Bibliography on power system probabilistic analysis (1962-1988)," IEEE Trans. Power Syst., vol. 5, pp. 1-11, Feb. 1990.

[8] R. Billinton and P. R. S. Kuruganty, "A probabilistic index for transient stability," IEEE Trans. Power App. Syst., vol. PAS-99, pp. 195-206, Jan./Feb. 1980.

[9] P. M. Anderson and A. Bose, "A probabilistic approach to power system stability analysis," IEEE Trans. Power App. Syst., vol. PAS-102, pp. 2430-2439, Aug. 1983.

[10] K. J. Timko, A. Bose, and P. M. Anderson, "Monte carlo simulation of power system stability," IEEE Trans. Power App. Syst., vol. PAS-102, pp. 3453-3459, Oct. 1983.

[11] F. F. Wu and Y.-K. Tsai, "Probabilistic dynamic security assessment of power systems," IEEE Trans. Circuits Syst. I, vol. CAS-30, pp. 143-159, Mar. 1983.

[12] A. M. L. da Silva, J. Endrenyi, and L. Wang, "Inegrated treatment of adequacy and security in bulk power system reliability evaluation," IEEE Trans. Power Syst., vol. 8, pp. 275-285, Feb. 1993.

[13] B. Porretta, D. L. Kiguel, G. A. Hamoud, and E. G. Neudorf, "A comprehensive approach for adequacy and security," IEEE Trans. Power Syst., vol. 6, pp. 433-441, May 1998.

[14] C. O. Nwankpa, S. M. Shahidehpour, and Z. Schuss, "A stochastic approach to small disturbance stability analysis," IEEE Trans. Power Syst., vol. 7, Apr. 1992.

[15] B. C. Lesieutre and J. R. Hockenberry, "Uncertainty analysis of power system simulations and ATC calculations using the probabilistic collocation method," in Bulk Power Systems Dynamics and Control IV-Restructuring, Santorini, Greece, Aug. 1998.

[16] I. A. Hiskens, M. A. Pai, and T. B. Nguyen, "Bounding uncertainty in power system dynamic simulations," in Proc. IEEE PES 2000 Winter Meeting, Singapore, Jan. 2000.

[17] M. A. Tatang, "Direct incorporation of uncertainty in chemical and environmental sSystems," Ph.D. dissertation, Massachusetts Inst. Technol., Cambridge, 1995.

[18] M. Webster, M. A. Tatang, and G. J. McRae, "Application of the probabilistic collocation method for an uncertainty analysis of a simple ocean model," Joint Program on the Science and Policy of Global Change, MIT, Cambridge, MA, Tech. Rep. 4, Jan. 1996.

[19] J. R. Hockenberry, "Evaluation of uncertainty in dynamic, reduced-order power system models," Ph.D. dissertation, Massachusetts Inst. Technol., Cambridge, Sept. 2000.

[20] P. J. Davis and P. Rabinowitz, Methods of Numerical Integration. New York: Academic, 1975.

[21] A. W. Drake, Fundamentals of Applied Probability Theory. New York: McGraw-Hill, 1967.

[22] P. Kundur, Power System Stability and Control. New York: McGrawHill, 1994. 
[23] J. V. Milanović and I. A. Hiskens, "Effects of load dynamics in power system damping," IEEE Trans. Power Syst., vol. 10, pp. 1022-1028, May 1995.

[24] I. A. Hiskens and J. V. Milanović, "Load modeling in studies of power system damping," IEEE Trans. Power Syst., vol. 10, pp. 1781-1788, Nov. 1995.

[25] Y. V. Makarov, V. A. Maslennikov, and D. J. Hill, "Revealing loads having the biggest influence on power system small disturbance stability," IEEE Trans. Power Syst., vol. 11, pp. 2018-2023, Nov. 1996.

[26] I. A. Hiskens and J. V. Milanović, "Locating dynamic loads which significantly influence damping," IEEE Trans. Power Syst., vol. 12, pp. 255-261, Feb. 1997.

[27] J. V. Milanović, I. A. Hiskens, and V. A. Maslennikov, "Ranking loads in power systems-Comparison of different approaches," IEEE Trans. Power Syst., vol. 14, pp. 614-619, May 1999.

[28] G. N. Ramaswamy, L. Rouco, O. Fillâtre, G. C. Verghese, P. Panciatici, B. C. Lesieutre, and D. Peltier, "Synchronic modal equivalencing (SME) for structure-preserving dynamic equivalents," IEEE Trans. Power Syst., vol. 11, pp. 19-29, Feb. 1996

[29] G. N. Ramaswamy, C. Evrard, G. C. Verghese, O. Fillâtre, and B. C. Lesieutre, "Extensions, simplifications, and tests of synchronic modal equivalencing (SME)," IEEE Trans. Power Syst., vol. 12, pp. 896-905, May 1997.

[30] G. N. Ramaswamy, "Modal structures and model reduction, with application to power system equivalencing," Ph.D. dissertation, Massachusetts Inst. Technol., Cambridge, June 1995.

[31] J. M. Hagee, "Explorations and extensions of synchronic modal equivalencing (SME)," Master's thesis, Massachusetts Inst. Technol., Cambridge, Feb. 1997.

[32] J. M. Hagee, B. Ayazifar, G. C. Verghese, and B. C. Lesieutre, "Load bus partitioning for synchronic modal equivalencing (SME)," in 28th North American Power Symp., Nov. 1996, pp. 57-61.

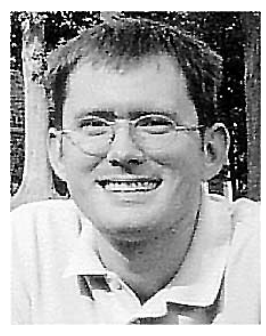

James R. Hockenberry (S'97-M'01) received the B.S. degree in engineering from Swarthmore College, Swarthmore, PA, in 1995, and the S.M. and $\mathrm{Ph} . \mathrm{D}$. degrees in electrical engineering from the Massachusetts Institute of Technology, Cambridge, in 1997 and 2000, respectively.

$\mathrm{He}$ is currently a Team Leader in electronics development at Robert Bosch, GmbH, Stuttgart, Germany.

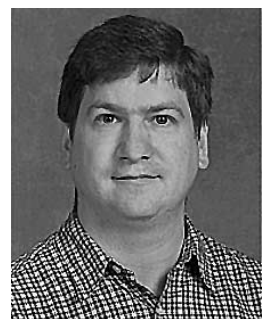

Bernard C. Lesieutre (M'04) received the B.S., M.S., and Ph.D. degrees in electrical engineering from the University of Illinois at Urbana-Champaign in 1986, 1988, and 1993, respectively.

From 1993 to 2001, he was an Assistant Professor and then Associate Professor of Electrical Engineering at the Massachusetts Institute of Technology, Cambridge, and a member of the Laboratory for Electromagnetic and Electronic Systems. He has served as Visiting Associate Professor at Cornell University, Ithaca, NY. He is presently a Staff Scientist at the Lawerence Berkeley National Laboratory, Berkeley, CA. 